\title{
CONTINUOUS DESCENT APPROACH: NOISE TEST FOR WARSAW INTERNATIONAL AIRPORT
}

\author{
Andrzej Gągorowski \\ Warsaw University of Technology \\ Faculty of Transport \\ Koszykowa Street 75, 00-662 Warsaw, Poland \\ tel.: +4822 2347337, fax: +48226292586 \\ e-mail:e-mail:agag@it.pw.edu.pl
}

\begin{abstract}
The noise generated by aircraft during departure and arrival flight operations continues to be significant problem at most major airports. Environmental impacts due to aviation operations in the vicinity of airports have become a serious concern to the community. A variety of techniques can be employed to reduce the noise impacts of aircraft. Continuous Descent Approach (CDA) is the new promising technique, which can significantly reduce noise impact by keeping aircraft higher and at lower engine thrust for longer by eliminating the level segments in conventional "step down" approaches. These procedures (implemented in different ways) are fixed routes that are vertically optimized. With the changing traffic, conditions and variable noise abatement rules the benefits of CDA operations are not yet fully realized. Actually, at many airports, CDA techniques are implemented for environmental improvements. This article presents the concept of optimized CDA. The modified flight procedure has been shown in measurement and analysis for Warsaw International Airport example. In this demonstration test, the procedure was shown to reduce the A-weighted noise level for the selected location along the flight path for different aircraft. Depending on aircraft type, the noise benefit from a CDA compared to a conventional approach was up to about 9.5 decibels (this change is noticeable to the human ear). Measurements have shown that Continuous Descent Approach have the potential for significant environmental benefits including reductions in noise.
\end{abstract}

Keywords: Continuous Descent Approach, CDA concept, aircraft type , air transport, demonstration test, noise pollution, noise environmental protection

\section{Introduction}

In the era of intensive development of air transport, noise reduction should be an integral part of the design process of aircrafts, routes, configurations and flight procedures. Developing quieter aircraft and improved techniques of flight operations is necessary to ensure air transport sustainable growth and thus protecting local communities and environment against adverse effects of noise. The Continuous Descent Approach (CDA) is the new promising technique, which can significantly reduce noise in the final phase of flight. Research at many airports confirms the effectiveness of this method in noise reductions[3, 4, 6]. However, CDA techniques can be implemented in different ways and that noise abatement can be different. Numerous research of CDA confirms different performance in this respect [1-4, 6, 9]. Variations in aircraft performance and complex airspace structures have limited widespread CDA implementation. For these above reasons, this method should be optimized.

The noise benefits for Continuous Descent Arrivals must be quantified in order to be adopted on a specified airport. Studies of Continuous Descent Approach at Warsaw International Airport have been conducted for several years by the Warsaw University of Technology team [10, 12] in cooperation with Polish Air Navigation Services Agency and Polish Airports. Author's goal of current research is development concept of CDA including different types of aircraft. The first phase of the study included the analysis of the noise caused by specified aircraft types performing the CDA procedures. 


\section{The general concept of optimized CDA}

The optimization of CDA procedures( implemented in different ways) are based usually on fixed routes (that are lateral or/and vertically optimized) [9]. This process involves many factors linking with environmental and weather conditions, arrival airspace characteristics( including lateral routing and altitude restrictions), aircraft performance and dynamics. Several trajectory optimization studies are performed and applied to specific arrivals [3, 4]. Noise-sensitive approach trajectory optimization for runway independent aircraft described in some studies [2]. Adaptative real-time optimization algorithm for noise abatement approach procedures was also proposed [13]. The dynamic optimization of trajectory is difficult nonlinear problem for solution involving also new navigation concepts.

A proposed concept of optimized CDA (Fig. 1) was developed to assist in the implementation of Continuous Descent Approach procedures designed to reduce noise under the flight path of aircraft during arrival operations at an airport. The arrival procedures using proposed concept CDA can be implemented for a single aircraft. The solution involves linking optimal trajectory (low noise flight profile) with specified aircraft types.

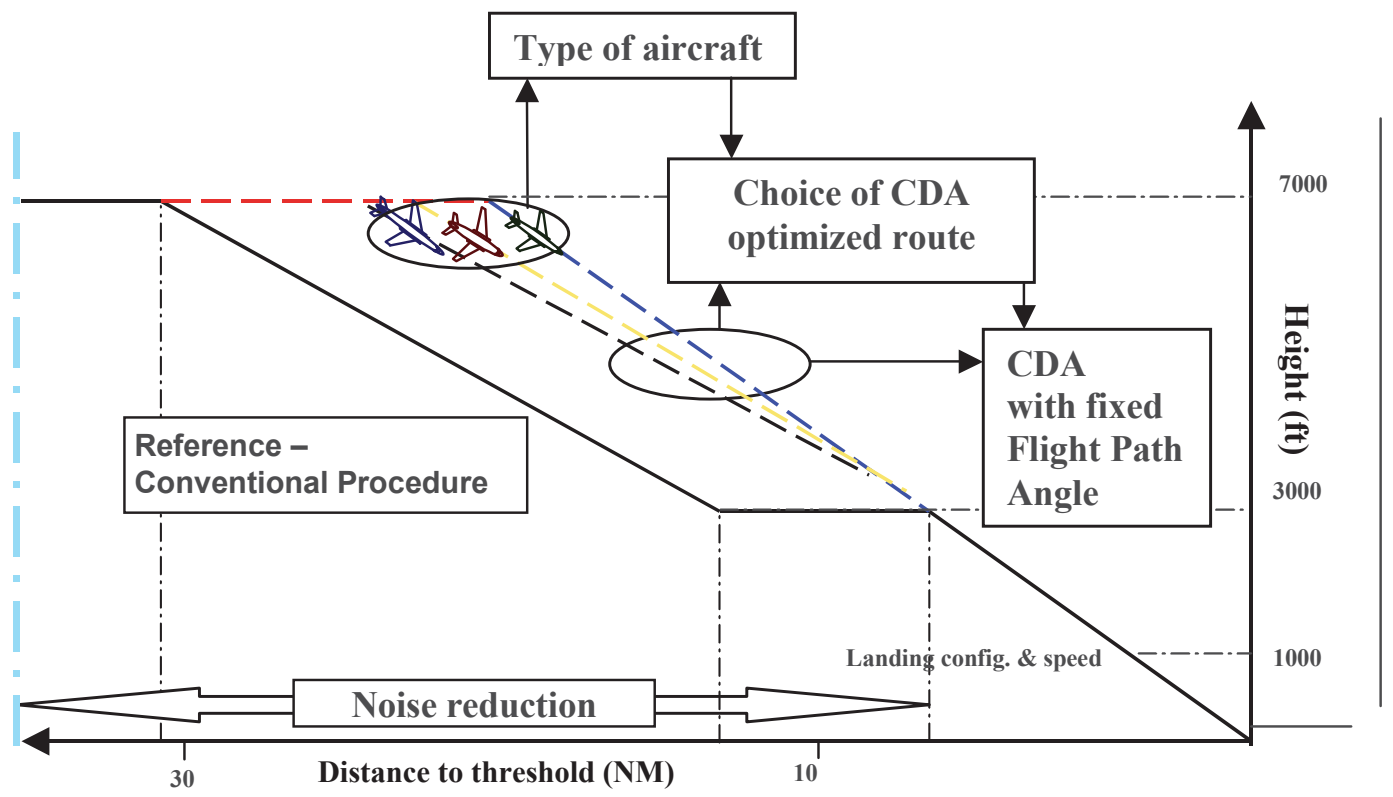

Fig. 1. The CDA concept

Research problem should be determined by defining and describing of selected indicators and parameters, which influence a basic traffic noise level, including:

- aircraft design parameters (as sound sources),

- operating parameters and performance of aircraft,

- parameters related to the construction of air transport infrastructure,

- environmental conditions,

- weather conditions.

For the algorithm design of optimized CDA, there is a need for the analysis of the parameters presented above. The main goal of this article is the noise emission study of aircraft with different design parameters.

\section{Noise test demonstration}

A mentioned early, noise test was conducted to demonstrate differences of the noise emission caused by specified aircraft types performing the CDA procedures. The airport chosen for this 
experiment was Warsaw Chopin Airport, with arrival to Runway 33, where most landing operations were performed [12]. In this experiment with different types of aircraft, requirements of national and international standards for measuring noise were included [5, 7, 8, 11]. The comparison with the Conventional Approach Descent also was conducted.

\subsection{Analysis of aircraft types}

Many different types of aircraft used in civil aviation have different state and configuration that makes noise assessment difficult in the flight operations. One of the major source noise components is airframe noise, which is a function of aircraft configuration-flap and landing gear position. Speed brake usage at low altitude increases airframe noise and should thus be minimized.

The Aircraft can be characterized by:

- state,

- configuration,

- aerodynamics,

- type, parameters, number and placement of engines,

- operating parameters and performance.

Tab. 1. Types of aircraft analysed in the study

\begin{tabular}{|c|c|c|c|c|c|}
\hline A/C Type & Airline & $\begin{array}{c}\text { Code } \\
\text { IATA/ICAO }\end{array}$ & A/C Type & Airline & Code IATA/ICAO \\
\hline A320 & Air France & AF/AFR & ATR72 & LOT Polish Airlines & LO/LOT \\
\hline A321 & Atlas Blue & 8A/BMM & E145 & LOT Polish Airlines & LO/LOT \\
\hline B737 & Aurela & -/LSK & ATR45 & LOT Polish Airlines & LO/LOT \\
\hline A320 & British Airways & BA/BAW & E175 & LOT Polish Airlines & LO/LOT \\
\hline A321 & British Airways & BA/BAW & B734 & LOT Polish Airlines & LO/LOT \\
\hline B735 & Czech Airlines & OK/CSA & B737 & Malév Hungarian Airlines & MA/MAH \\
\hline B738 & Eurocypria & UI/ECA & B738 & Malév Hungarian Airlines & MA/MAH \\
\hline E170 & Finair & AY/FIN & B733 & Norwegian Air Shuttle & DY/NAX \\
\hline E190 & Finair & AY/FIN & B737 & Norwegian Air Shuttle & DY/NAX \\
\hline A320 & Finair & AY/FIN & B734 & Sky Airlines & ZY/SHY \\
\hline E190 & Finair & AY/FIN & A321 & Sky Airlines & ZY/SHY \\
\hline E190 & KLM Dutch Airlines & KL/KLM & B738 & Sky Airlines & ZY/SHY \\
\hline B733 & Lufthansa & LH/DLH & B738 & Travel Service & QS/TVS \\
\hline E190 & Lufthansa & LH/DLH & A320 & Tunisair & TU/TAR \\
\hline B734 & LOT Polish Airlines & LO/LOT & MD11F & United Parcel Service & 5X/UPS \\
\hline E170 & LOT Polish Airlines & LO/LOT & \multirow{2}{*}{ A320 } & Wizz Air Hungary & W6/WZZ \\
\hline B735 & LOT Polish Airlines & LO/LOT & & & \\
\cline { 1 - 2 } & & & & & \\
\end{tabular}

Table 1 presents the types of aircraft, which the noise level has been analysed in the study. Among them, we can distinguish the follow major groups:

- Embraer E-Jets family (E-170/E-175/E-190/E-195) - a series of narrow-body(a single aisle aircraft), twin-engine, medium-range jet airliners,

- Airbus A320 family (A320, A321)- consisting of short- to medium-range, narrow-body, commercial passenger jet airliners (low-wing cantilever monoplanes with a conventional tail unit with a single vertical stabilizer, rudder and high-bypass turbofan engines),

- Boeing 737 Classic variants (B733/B734/B735/ B736/ B737) - a short- to medium-range twinengine narrow-body jet airliners (the 737 Classic series /300, 400, 500/ featured CFM56 turbofan engines, which yielded significant gains in fuel economy and a reduction in noise),

- Boeing 737 Next Generation (B738/B739) improved narrow-body jet airliners (e.g. controlled engines CFM56-7, 2.5deg nozzle tilt, redesigned struts, improved nacelles with increased airflow and improved noise treatment), 
- McDonnell Douglas MD-11F (cargo version) - a three-engine medium- to long-range widebody jet airliner (it features a stretched fuselage, increased wingspan with winglets, refined airfoils on the wing and smaller tailplane. Two of its engines are mounted on underwing pylons and a third engine at the base of the vertical stabilizer),

- ATR series (ATR45, ATR72) - a twin-engine turboprop (turbine engine which drives an aircraft propeller using a reduction gear) short-haul regional airliner.

\subsection{Measurement procedures}

The noise levels were determined in accordance with national and international standards and regulations. (ICAO recommendations [7], ISO-196-1 [8], and Regulation of Polish Environment Minister [11]). ISO 1996-1:2003 defines the basic quantities to be used for the description of noise in community environments and describes basic assessment procedures. Regulation of Polish Environment Minister specifies methods to assess environmental air noise.

The main noise measurement points were located on the extended centreline of the runway 33 . Fig. 2 shows sample paths of aircraft approaching for landing. Fig. 2 illustrates also the sample measurement point localised on the extended centreline of the runway $9.72 \mathrm{NM}$ from the threshold and near the point of navigation - MASAK. On level ground, this corresponds to a position vertically below the $3^{\circ}$ descent path(FPA).

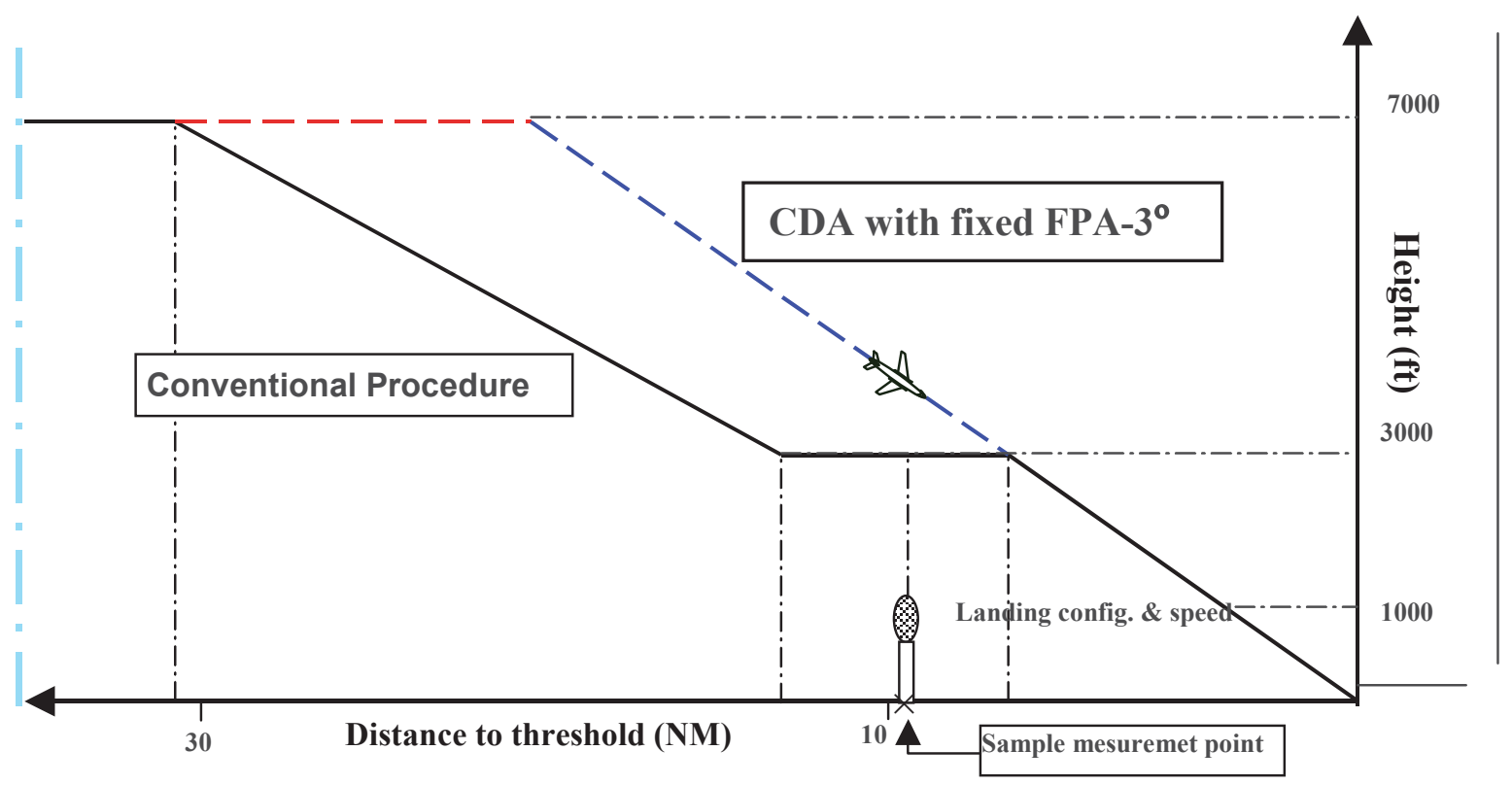

Fig. 2. The sample location of measurement point relative to CDA and Conventional Path

Aircraft sound measurements generally use the metric known as A-weighted sound level. The effect of noise exposure during an acoustic event can be described as the sound exposure level (SEL, $L_{A E}$ ) of individual aircraft noise events:

$$
L_{A E}=S E L=10 \cdot \log \left[\frac{1}{t_{o d}} \int_{t_{0}}^{t_{1}} 10^{0,1 * L_{A}(t)} d t\right], d B,
$$

where:

$L_{A}-\mathrm{A}$-weighted instantaneous sound pressure level $[\mathrm{dB}(\mathrm{A})]$,

$t_{o d}$ - referenced time,

$t_{0}$ - start time,

$t_{1}-$ end time. 
The sound exposure level (referenced to a standard period $t_{o d}$ of 1 second) represents the cumulative sound energy detected above an established threshold for a single event considering both intensity and duration of the sound. The SEL for each event was calculated using the conventional method of logarithmic summation of recorded noise levels between the two instances(before and after the peak level that are $10 \mathrm{~dB}$ lower than the peak level). After the noise events (correlated to aircraft flights), the time histories were screened to determine the A-weighted noise level for each noise event. For the purposes of this analysis, the measurement data were arithmetically averaged to obtain the mean noise level by aircraft type for each monitor location.

\section{Analysis and results}

The analysis was conducted for the most advanced commercial airplanes including the Airbus series, and the Boeing family, and MD-11 (they are described in Chapter 3.1) at the similar weather and operating conditions, and for the same configuration. The assessment of aircraft noise was made by calculating the average sound exposure level $\left(L_{A E}\right)$ for all events of the same aircraft type and regarding the standard deviation with 95 percent confidence interval. Average $L_{A E}$ can be evaluated from the dependence (2):

$$
L_{A E}=10 \cdot \log \left(\frac{1}{k} \sum_{j=1}^{k} 10^{0,1 L_{A E j}}\right),
$$

where:

$k-$ current number of aircraft operations

$L_{A E j}$ - the sound exposure level of single individual aircraft noise event [dB]

The standard deviation can be described as follows:

$$
\sigma_{A E}=\sqrt{\frac{1}{k-1} \sum_{j=k}^{k}\left(10^{0,1 L_{A E j}}-E_{A}\right)^{2}}
$$

where $E_{A}-$ value of average noise exposure expressed as:

$$
E_{\mathrm{A}}=\frac{1}{k} \sum_{j=1}^{k} 10^{0,1 L_{A E j}} .
$$

The differences of noise emission are very large between analysed aircrafts. For the analysed aircrafts, the average sound exposure level $\left(L_{A E}\right)$ ranged between 63.9 and $85.5 \mathrm{dBA}$, with a standard error up to $1.7 \mathrm{dBA}$. This result is very significant if we take into consideration the types of aircraft only. As expected, the values for noise measures decrease with increasing distance from the runway. Additionally, for the same aircraft types, but different operators(airlines) some of the variations in the noise levels are due to mass, flap changes, engine thrust transients, and/or difference in site elevations.

The average $L_{A E}(S E L)$ noise levels at the sample measurement site (presented in Fig. 2) are shown in Fig. 3 for selected aircrafts that performed the CDA (at Height of 3000 feet). For the examples the average $L_{A E}$ ranged between 71.9(E145 of Polish Airlines) to $81.9 \mathrm{dBA}$ (MD11 of UPS airline), with a standard error up to $0.3 \mathrm{dBA}$.

A complete comparison of the noise from aircraft performing the CDA to aircraft performing the Conventional Approach showed that the noise reduction is significant and also depended from types of aircraft. The reduction of the A-weighted noise level at locations along the flight path was by 0.9 to $9.5 \mathrm{dBA}$. The differences of values for noise measures decrease with decreasing distance to the runway threshold (and thus altitude). A comparison of sample results for the Baseline, Standard Approach(STD), and CDA for selected types of aircraft shown in Fig. 4. For this examples, the noise benefits from a CDA compared to a Conventional Approach are from 3.1 (MD11 of UPS airline) to 8.3 decibels(B735 of Czech Airlines) depending on the type of aircraft. 


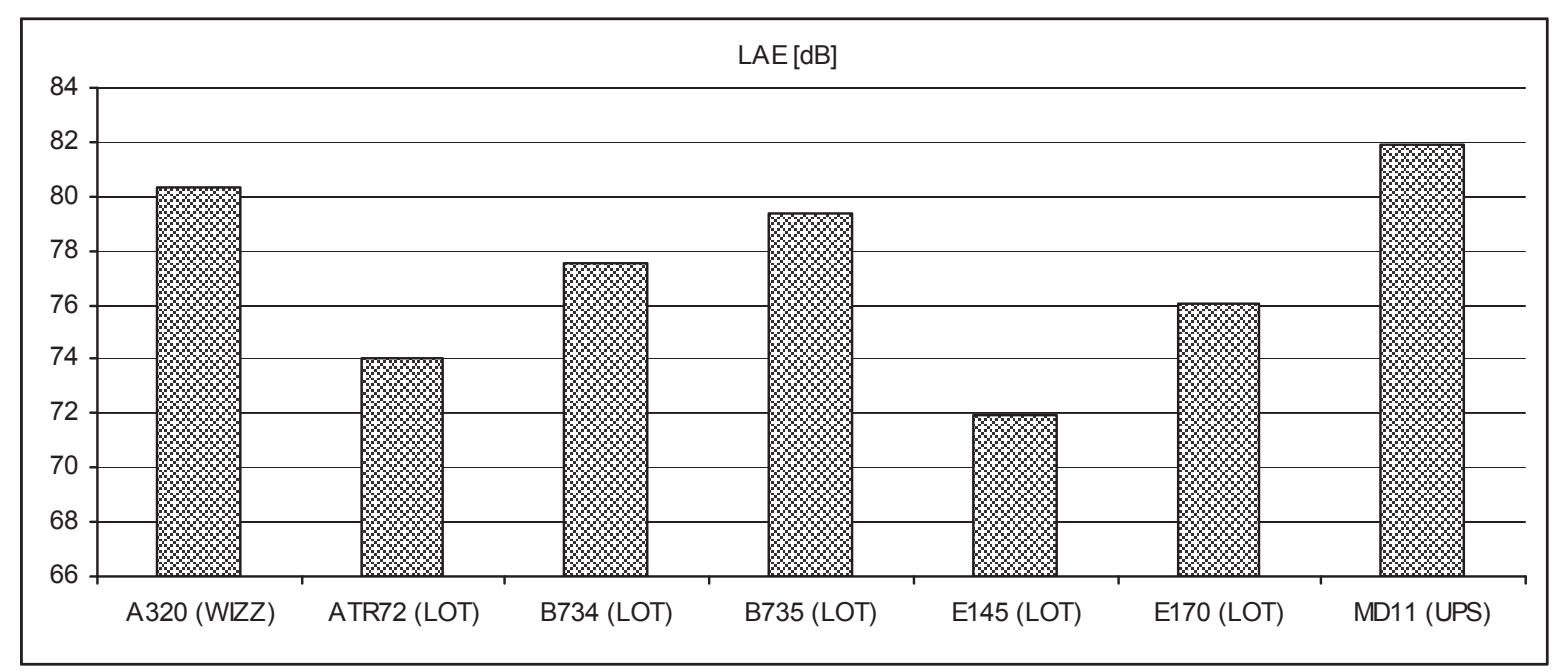

Fig. 3. The example of average sound exposure levels emitted by the different types of aircrafts

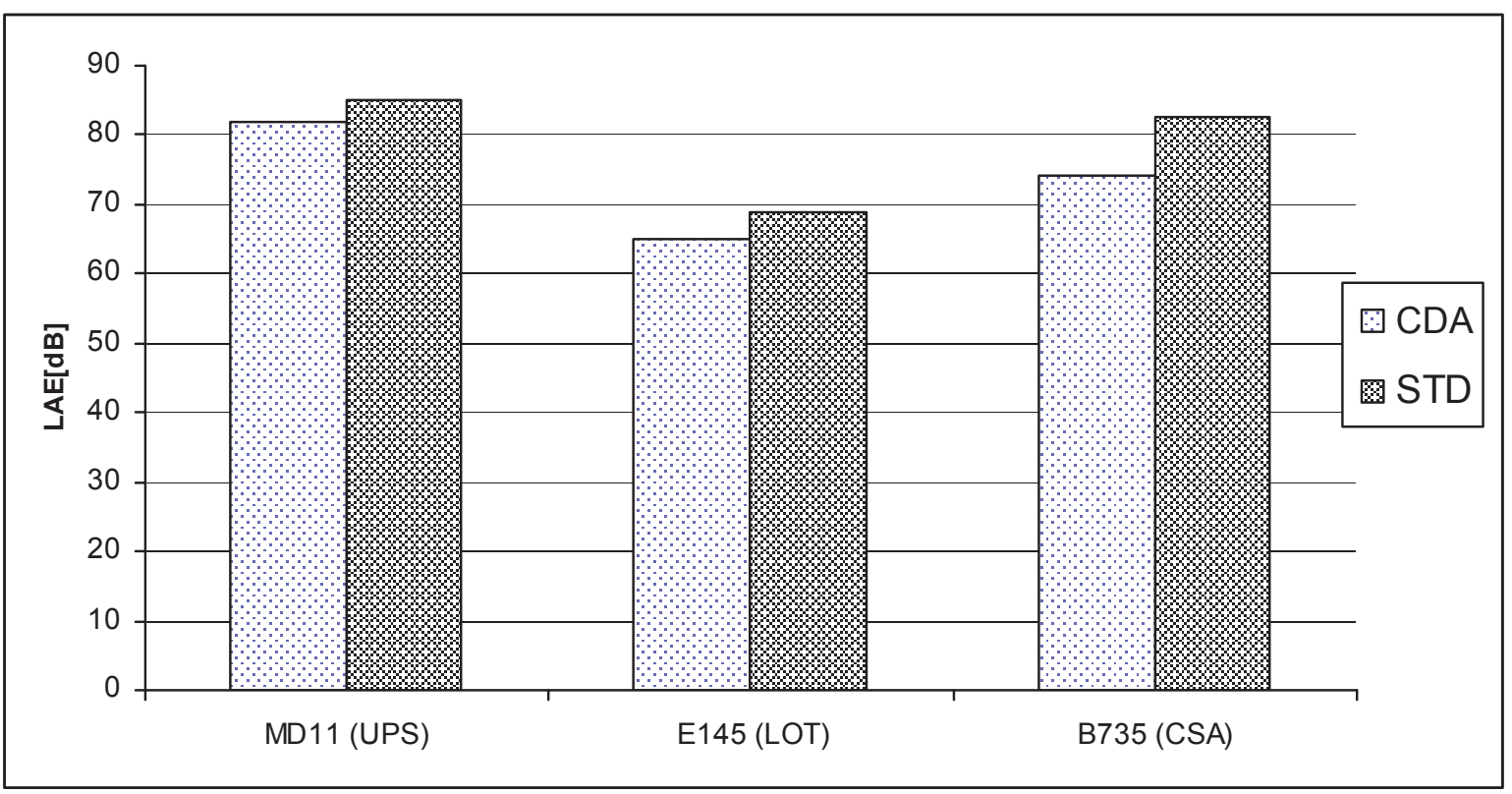

Fig. 4. The sample comparison of CDA and Standard Approach for SEL noise emitted by the different aircrafts

From these results, it can be concluded that the large differences and variations between the types of aircraft translate into the level of noise emission. The analysis showed that the aircraft type should be included to define optimal trajectory (low noise flight profile) during arrival operations.

\section{Conclusions}

In this paper, the general concept of optimized CDA has been proposed to provide a low noise at high-efficiency continuous descent profile. The solution conditions a choice of descent profile by specified aircraft type. In demonstration test, the procedure was shown to reduce the noise level at the selected location along the flight path for different aircraft. The goals of the analysis presented in this paper were shown differences of the noise emission caused by specified aircraft types performing the CDA procedures. The study was conducted for the most advanced commercial airplanes including the Airbus series, and the Boeing series, and MD-11. 
The analysis presented in this paper show that the level of noise reduction is significant depended on the type of aircraft. It is therefore necessary to include the aircrafts parameters along with a wide environmental conditions and arrival airspace characteristics in design process of variants of the CDA approaches. The CDA optimisation will allow for better improvement of environmental impact. This will provide noise relief to communities around airports during from arriving aircraft and compliance with the limit values.

The comparison with the conventional approach descent also was conducted. For example, they have been used during operations at Warsaw Airport can reduce noise by up to 9.5. CDA's have the potential for reducing noise, but these benefits must be quantified in order to be adopted on a broad scale. Variations in types and performance aircraft and complex airspace structures can have limited widespread CDA implementation.

The results indicate the need for further research on CDA methods. In next steps will be determined which factors have the greatest impact on the level of air traffic noise. This will define a set of assumptions (guidelines) needed to develop a comprehensive method of CDA optimization in terms of noise reduction.

\section{References}

[1] Alam, S., Nguyen, M. H., Abbass, H. A., Lokan, Ellejmi, M., Kirby, S., A dynamic continuous descent approach methodology for low noise and emission, 28th Digital Avionics System Conference (DASC), pp. 1-18, Salt Lake City, Utah, USA 2010.

[2] Atkins, Ella M., Xue, Min., Noise-sensitive final approach trajectory optimization for runway independent aircraft, Journal of aerospace computing, information and communication, 1(7), pp. 269-287, 2004.

[3] Clarke, J.-P. B., Ho., N. T., Ren, L., Brown, J. A., Elmer, K. R., Tong, K.-O., Wat, J. K., Continuous Descent Arrival: Design and Flight Test for Louisville International Airport, AIAA Journal of Aircraft, Vol. 41, No. 5, pp. 1054-1066, 2004.

[4] Clarke, J.-P., Bennett, D., Elmer, K., Firth, J., Hilb, R., Ho, N., Johnson, S., Lau, S., Ren, L., Senechal, D., Sizov, N., Slattery, R., Tong, K.-O., Walton, J., Willgruber, A., Williams, D., Development, design, and flight test evaluation of a continuous descent Arrival procedure for nighttime operation at Louisville International Airport, Report PARTNER-COE-2006-002, 2006.

[5] European Civil Aviation Conference, Report on Standard Method of Computing Noise Contours around Civil Airports, ECAC.CAEC Doc 29, Third Edition, 2006.

[6] International Civil Aviation Organization(ICAO), Review of noise abatement procedure research \& development and implementation results discussion of survey results, preliminary edition, 2007.

[7] International Civil Aviation Organization(ICAO), Environmental protection- Vol. I -aircraft noise, Annex 16, International Standards and Recommennded Practices, fifth edition, 2008.

[8] ISO 1996-1:2003, Acoustics -- Description, measurement and assessment of environmental noise - Part 1: Basic quantities and assessment procedures, 2003.

[9] Lenz, H., Korn, B., Enabling advanced continuous descent approaches -results of the european project optimal, Digital Avionics Systems Conference, IEEE/AIAA 28th, pp. 2.C.3-1 - 2.C.3-10, DASC 2009.

[10] Nowecki, M., Comparative analysis of aircraft noise according to the procedures of classical and smooth approach of civil aircrafts at the airport Warsaw -Okecie, Master Thesis, (thesis supervisor, Gągorowski A.), Warsaw University of Technology, Faculty of Transport, Warsaw 2011.

[11] Polish Ministry of the Environment, Regulation of Environment Minister dated 2 October 2007 on requirements for emission measurements of substances or energy in the environment by managing road, tram lines, airport, port, 2007. 
[12] Toruński, K., A noise measurement and assessment method for the purpose of a comparative analysis of standard and continuous aircraft approach procedures, Master Thesis, (thesis supervisor, Gągorowski A.), Warsaw University of Technology, Faculty of Transport, Warsaw 2011.

[13] Zou, K. F., Clarke, J.-P, Adaptative real-time optimization algorithm for noise abatement approach procedures, Proceedings of the 3rd AIAA aviation technology, integration, and operations (ATIO) conference, Vol. 1, AIAA paper No 2003-6771, Denver, Colorado, USA 2003. 